\title{
SEIZURE-ALERTING DOGS IN EPILEPSY MANAGEMENT
}

Families of all children ( 1 to 18 years old) followed at the Refractory Epilepsy Clinic, Alberta Children's Hospital, Canada, were surveyed and those who had a dog for at least one year while having at least 1 seizure/month were selected and questioned regarding seizurerelated behaviors. Forty-eight families (39\%) owned a dog, and twenty families (42\%) reported specific seizure-related behaviors by their dog during or immediately after the child's seizure. Most seizure-response dogs (SRDs) $(68 \%)$ were larger animals ( $>40 \mathrm{lbs}$ ), mostly mixed breeds. Response behaviors included licking of the face, decreased motor activity, and whimpering, usually during or after the event. Anticipatory behavior, preceding the seizure by 2.5 minutes (range, $10 \mathrm{sec}$ to 5 hours), occurred in $41 \%$ of the SRDs (approximately $15 \%$ of all dogs studied). The seizure-alerting dogs (SADs) were mainly female $(80 \%)$, larger animals, including Golden Retriever, Standard Poodle, and German Shepherd. The alerting behaviors were thought to represent a unique sensory ability; they usually existed with the first seizure, were seizure type and child specific, had a median sensitivity estimate of $80 \%$, and they never occurred without a subsequent seizure. They were protective in nature (eg the dog would sit on a toddler prior to an expected drop attack), and no instance of aggression was reported. Quality of life was higher in families with a dog, and particularly if the dog was seizure-responsive. (Kirton A, Wirrell E, Zhang J, Hamiwka L. Seizure-alerting and -response behaviors in dogs living with epileptic children. Neurology June (2 of 2) 2004;62:2303-2305). (Reprints: Dr A Kirton, Division of Pediatric Neurology, Department of Pediatrics, Alberta Children's Hospital, 1820 Richard Rd SW, Calgary, AB T2T 5C7, Canada).

COMMENT. Perhaps the earliest examples of the therapeutic value and protective instinct of dogs are recorded in Elizabethan times, when the Cavalier King Charles Spaniel, often depicted in royal portraiture, is named 'Comforter' and is credited with "strange healing powers" (1570) by Dr Johannes Caius, physician to the Queen. The dog is found under the skirts of Mary Queen of Scots at her beheading in 1563, at the feet of Charles I when beheaded in 1649, and the constant companion of Charles II, 1660-85 (Forword M. The Cavalier King Charles Spaniel. London, Hutchinson, 1980). The Cavalier King Charles Spaniel should be added to the list of breeds likely to have anticipatory seizure-responsive behavior in future efforts to validate these canine instincts.

\section{FAMILIAL EPISODIC AMAUROSIS}

A family with a stereotyped unilateral or bilateral transient visual loss, that recurred many times daily and was associated with childhood epilepsy and familial hemiplegic migraine, is reported from University Hospitals, Geneva, Switzerland. The index case was a 43-year-old man who had 3 to 10 daily episodes since infancy, commonly provoked by sudden changes of light intensity or by pressure on the eyelids, and followed by a $30 \mathrm{sec}$ refractory period when amaurosis could not be reprovoked. Illumination of the affected eye in unilateral amaurosis failed to induce pupillary responses, whereas the response to illumination of the unaffected eye was normal, with both direct and consensual pupillary constriction. Between episodes, pupillary responses and the neurologic exam, including MRI 